\title{
Surface anatomy and prevalence of fibularis tertius muscle in a south-western Nigerian population
}

\author{
James Olumide Ashaolu ${ }^{1 *}$, Oluwaseun Iretiolu Olorunyomi ${ }^{1}$, Oluwole Adebayo Opabunmi ${ }^{2}$, \\ Victor Okoliko Ukwenya ${ }^{1}$, Magnus Adebo Thomas ${ }^{1}$ \\ ${ }^{1}$ Department of Anatomy, Bowen University, Iwo, Nigeria; *Corresponding Author: Olusco172003@yahoo.com \\ ${ }^{2}$ Department of Physiology, Bowen University, Iwo, Nigeria
}

Received 25 December 2012; revised 28 January 2013; accepted 16 February 2013

Copyright (c) 2013 James Olumide Ashaolu et al. This is an open access article distributed under the Creative Commons Attribution License, which permits unrestricted use, distribution, and reproduction in any medium, provided the original work is properly cited.

\begin{abstract}
Fibularis tertius is a variant muscle of the leg that inserts in the foot. It has been implicated in providing clues to the evolution of upright posture in man. This work investigates the prevalence of fibularis tertius muscle in the African population, using south-western Nigeria as a sample population. The percentage prevalence of fibularis tertius muscle in the population studied is $63 \%$, with higher prevalence in males compared to females. The muscle was however observed to present itself more on the right limb than on the left. Fifteen percent $(15 \%)$ of the subjects had fibularis tertius muscle only on the right foot. Six percent $(6 \%)$ had the muscle on the left foot only, $52 \%$ had it on both feet and $27 \%$ did not have the muscle on any of their foot. A unique surface positioning of the fibularis tertius tendon was also observed in all the subjects studied. These findings may provide contributions to the clinical evaluation of the muscle.
\end{abstract}

Keywords: Fibularis Tertius Muscle; Variant Muscles; Foot

\section{INTRODUCTION}

Unfolding the evolutionary stance of the fibularis tertius muscle (FTM) is still a current quest. The muscle is absent in hominoid apes and with the acquisition of the bipedal gait; the muscle emerged as a recent addition in the human foot [1]. The muscle has attracted different definitions recently, while different investigators have discussed its nature in the light of clinical assessment, evolution of upright standing, bipedalism and ankle injury. Phylogenetically, the human FTM is relatively a young structure with various modes of insertions sug- gesting a recent development in its ontogeny. FTM fibres arise from the distal third or more of the medial surface of the fibula and the adjoining anterior crural intermuscular septum. The tendon passes behind the superior extensor retinaculum that it shares with extensor digitorum longus. It is reported to usually insert into the medial part of the dorsal surface of the base of the fifth metatarsal bone. Various patterns of insertion have been reported [2].

Although, FTM acts along with extensor digitorum longus and tibialis anterior as a dorsiflexor of the foot, it also acts with fibularis longus and brevis muscles as a strong evertor of the foot and prevents hyper-inversion. It has been suggested that FTM helps in transmitting body weight to the lateral longitudinal arch during the stance phase of walking and gives helpful contribution to the swing phase of bipedal mode of locomotion [3,4].

Currently, the muscle is known to be a variant muscle of the leg and its percentage prevalence is not yet established in most populations. The Bolivian population has presented with the highest prevalence (100\%) [5], while the Jewish population presented with the least (10\%) [6]. No previous study conducted on an African population was found. Amassing information on the nature and prevalence of the muscle in different populations may deepen insight into the evolutional progression of the muscle and help in the clinical evaluation of the muscle in the populations with established prevalence.

However, the knowledge of the actual surface palpation and somewhat unique anatomy of FTM may be of importance to clinicians, foot surgeons, demonstrators, and towards successful tissue grafting procedures. Surface palpation of the FTM has been demonstrated by other investigators and confirmed to have $100 \%$ level of accuracy compared with magnetic resonance imaging studies (MRI) [4].

The pull of the FTM may be responsible for causing stress on the fifth metatarsal and account for all stress fractures in any individual [3]. However, the contrac- 
tions of the FTM may be responsible for delayed healing of the fifth metatarsal shaft fracture [7].

Against this background, the purpose of this study was to examine the prevalence of FTM in an African population of the South-western region of Nigeria through a surface evaluation of the foot of young adult individuals.

\section{MATERIALS AND METHODS}

\subsection{Source of Subjects}

This research was conducted in the Faculty of Basic Medical Sciences, College of Health Sciences, Bowen University, Iwo, Osun State, Nigeria, using the limbs of 100 medical students (53 females and 47 males) which correspond to 200 feet in all. The average age of the subjects was 20.5 years, with upper and lower limits of 16 and 25 years, respectively. The study was conducted between the months of February and March, 2012. Ethical clearance was obtained from the College of Health Sciences Committee on Research Ethics, Bowen University and experimental procedures conformed to the guide by the US Department of Health and Human Services.

\subsection{Techniques of Data Collection}

Tribe, gender, age, presence of fibularis tertius muscle on each limbs (left and right), past record of ankle injuries in both limbs, the degree to which the muscle can be palpated was recorded for each subject examined.

\subsection{Subject Evaluation Procedure}

Fibularis tertius muscle is clearly identifiable on the dorsum of the foot by its tendon when present, thus evaluation techniques described by Tixa [6] and Kendal et al. [8] were adopted and slightly modified in this study. Each foot of the subjects was doubly examined for 80 seconds by two evaluators. Tixa [6] and Kendall et al. [8] had assessed the ankle for the presence of fibularis tertius muscle. But, in an unpublished pilot investigation we had conducted, the fibularis tertius muscle was rather palpable in the dorsal surface of the feet of the subjects, lateral to the last tendon of extensor digitorum brevis.

In this study, the subjects were seated and their knees were flexed at approximately $110^{\circ}$. The evaluators were squatting in front of the subjects fixing their legs on the ankle joint.

The degree to which the muscle could be seen was designated:

G1-When no muscular tension was needed to be applied before the muscle could be seen.

G2-When dorsiflexion and eversion of the foot were employed before the muscle could be seen.

G3 - When dorsiflexion and eversion of the foot were employed against a resistance before the muscle is seen.
G3 is an assessment protocol progression that reveals the outline of the fibularis tertius muscle when present and all the other muscle tendon of the foot.

To ascertain the presence of the muscle, the following palpation procedures were engaged:

Step 1: The last tendon of extensor digitorum longus was palpated on its way from the little toe to the inferior extensor retinaculum;

Step 2: The subject dorsiflexes and everts the specific foot. Surface bulging of the extensor digitorum brevis muscle was identified;

Step 3: The surface groove between the last tendon of extensor digitorum longus muscle and the bulging of extensor digitorum brevis was palpated to locate the fibularis tertius muscle.

\subsection{Method of Data Analysis}

The data collected was processed by simple analytical methods involving the determination of frequency and percentage occurrences of the fibularis tertius muscle.

\section{RESULTS}

Out of the 200 legs studied, FTM was found present on 67 right legs and on 58 left legs making a sum total of 125 occurrences (Table 1). Therefore, the percentage prevalence of FTM equals $63 \%$ of the sample population studied (Figure 1). The total number of male limbs studied is ninety-four ( $\mathrm{n}=94)$. FTM was present on thirty-three (33) right limbs and twenty-nine (29) left

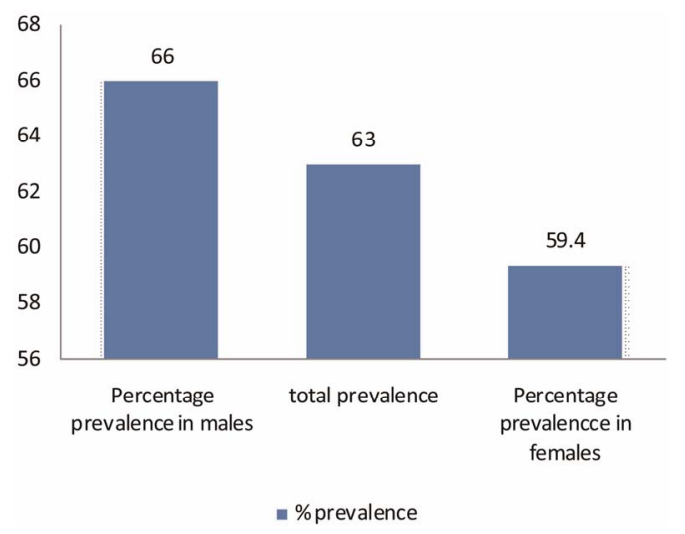

Figure 1. Percentage prevalence in males, all studied subject and in females.

Table 1. Prevalence of fibularis tertius in each limb of 100 subjects examined.

\begin{tabular}{cccc}
\hline \multicolumn{2}{c}{ Right Legs $(\mathrm{n}=100)$} & \multicolumn{2}{c}{ Left Legs $(\mathrm{n}=100)$} \\
\hline Present & Absent & Present & Absent \\
$\mathrm{n}=67$ & $\mathrm{n}=33$ & $\mathrm{n}=58$ & $\mathrm{n}=42$ \\
$67 \%$ & $33 \%$ & $58 \%$ & $42 \%$ \\
\hline
\end{tabular}


limbs. So, fibularis tertius prevalence in the studied male subjects is 66\% (Table 1 and Figure 1). Amongst the 106 female subjects studied right leg with fibularis tertius present is thirty-four (34) while left legs with fibularis tertius present is twenty-nine (29). FTM prevalence in the female subjects is 59.4\% (Table 1 and Figure 1).

On the right foot, fibularis tertius is commoner in females $(51 \%)$ than in males (49\%). Both of equal prevalence on the left foot, being (50\%) in males and females (Table 2 and Figure 2). 15\% of the subjects had fibularis tertius muscle only on the right foot. $6 \%$ had the muscle on the left foot only, 52\% had it on both feet and $27 \%$ did not have the muscle on any of their foot (Table $\mathbf{3}$ and Figure 3).

The total number of ankle injuries occurring in 200 limbs of the 100 subject samples studied is 23 . 15 past ankle injuries were recorded on the right foot of the 100 subjects examined, 9 of those 15 had the muscle and 6 did not. On the left foot, 8 subjects had a record of past

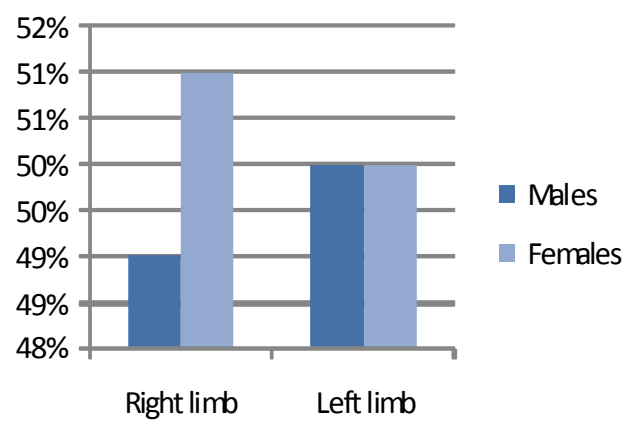

Figure 2. Percentage occurrence of fibularis tertius muscle in right and left limbs in males and females.

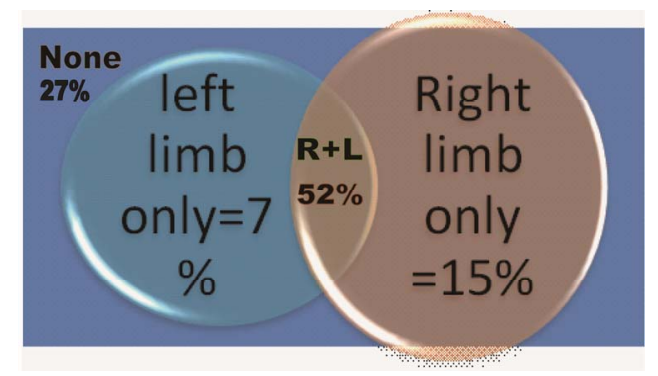

Figure 3. Individual limb distribution of fibularis tertius in the subjects studied. $\mathrm{R}+\mathrm{L}$ - subjects with FTM on both right and left limbs, None-subjects without F-TM on either feet.

Table 2. Gender variations of the prevalence of fibularis tertius.

\begin{tabular}{ccccc}
\hline Right & \multicolumn{2}{c}{ Limb $(\mathrm{n}=67)$} & \multicolumn{2}{c}{ Left Limb $(\mathrm{n}=58)$} \\
\hline $\begin{array}{c}\text { Sex } \\
\text { Number }\end{array}$ & Males & Females & Males & Females \\
$\begin{array}{c}(\mathrm{n}=33) \\
\text { Percentage }\end{array}$ & $49 \%$ & $51 \%$ & $50 \%$ & $50 \%$ \\
\hline
\end{tabular}

ankle injuries. 5 of those 8 had the muscle and 3 did not (Table 4). However, throughout the procedures of palpation, 3 cases were recorded with the G1, 41 cases were recorded with the G2 and 81 cases with the G3 (as seen in Table 5)

Figures 4 and 6 gave illustrations of the observed relatively medial palpation site of fibularis tertius tendon (as we have observed in our subjects) when compared to more lateral location in Figure 5 (as classically described in textbooks).

Table 3. Percentage limb distribution of fibularis tertius amongst the 100 subjects studied.

\begin{tabular}{cccc}
\hline $\begin{array}{c}\text { Presence on } \\
\text { right limb only }\end{array}$ & $\begin{array}{c}\text { Presence on } \\
\text { left limb only }\end{array}$ & $\begin{array}{c}\text { Presence in } \\
\text { both limbs }\end{array}$ & $\begin{array}{c}\text { Absence in } \\
\text { both limbs }\end{array}$ \\
\hline $\begin{array}{c}15 \text { cases } \\
15 \%\end{array}$ & 6 cases & 52 cases & 27 cases \\
$6 \%$ & $52 \%$ & $27 \%$ \\
\hline
\end{tabular}

Table 4. FTM variation in relation to ankle injury.

\begin{tabular}{ccccc}
\hline & $\begin{array}{c}\text { Number of } \\
\text { ankle injuries } \\
\text { occurring }\end{array}$ & $\begin{array}{c}\text { \% Ankle } \\
\text { injury } \\
\text { occurrence }\end{array}$ & $\begin{array}{c}\text { Fibularis } \\
\text { tertius } \\
\text { presence }\end{array}$ & $\begin{array}{c}\text { \% Fibularis } \\
\text { tertius } \\
\text { occurrence }\end{array}$ \\
\hline $\begin{array}{c}\text { Right } \\
\text { limbs } \\
\begin{array}{c}\text { Left } \\
\text { limbs }\end{array}\end{array}$ & 15 & $65 \%$ & 9 & $60 \%$ \\
\hline
\end{tabular}

Total number of Ankle Injuries occurring = 23 .

Table 5. Graded series (G1, G2, G3) indicating the ease of palpation of fibularis tertius muscle in each limb.

\begin{tabular}{ccccc}
\hline & $\begin{array}{c}\text { Number } \\
\text { of G1 } \\
\text { cases }\end{array}$ & $\begin{array}{c}\text { Number } \\
\text { of G2 } \\
\text { cases }\end{array}$ & $\begin{array}{c}\text { Number } \\
\text { of G3 } \\
\text { cases }\end{array}$ & $\begin{array}{c}\text { Total cases } \\
\text { with FTM } \\
\text { present }\end{array}$ \\
\hline Right Legs & 2 & 20 & 45 & 67 \\
Left Legs & 1 & 21 & 36 & 58 \\
\hline
\end{tabular}

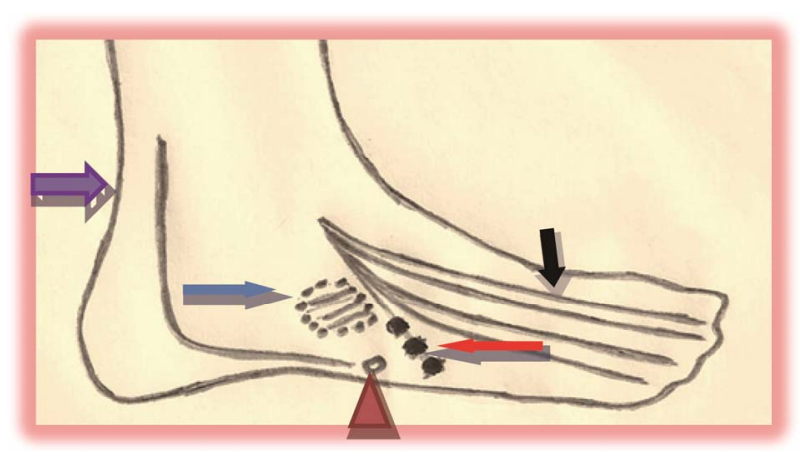

Figure 4. Showing the dorsal-lateral surface of the foot in the studied population.Purple arrow-fibularis brevis tendon; Blue arrow-extensor digitorum brevis muscle; Black arrow-extensor digitorum longus tendon; Orange arrow-fibularis tertius tendon; Brown arrow head—base of fifth metatarsal bone. 


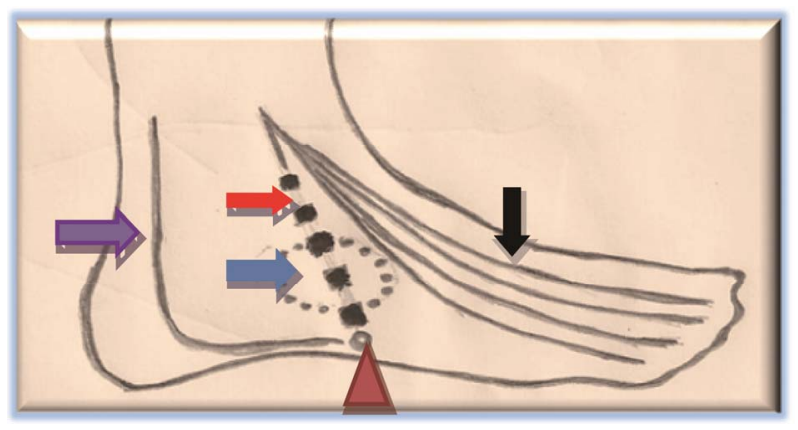

Figure 5. Showing the dorsal-lateral surface of the foot in usual textbook pattern.Purple arrow-fibularis brevis tendon; Blue arrow-extensor digitorum brevis muscle; Black arrowextensor digitorum longus tendon; Orange arrow-fibularis tertius tendon; Brown arrow head-base of fifth metatarsal bone.

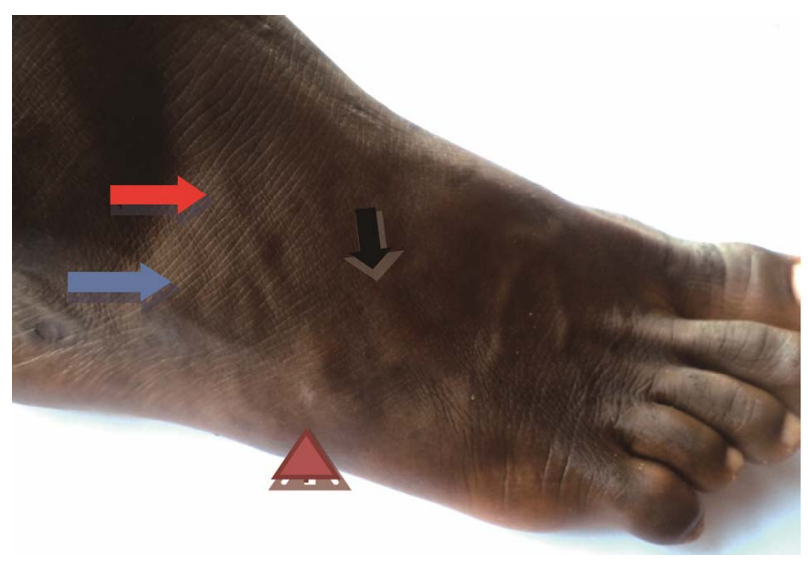

Figure 6. Showing the dorsal-lateral surface of the foot in living human foot. Blue arrow-extensor digitorum brevis muscle; Black arrow-extensor digitorum longus tendon; Orange arrow-fibularis tertius tendon; Brown arrow head-base of fifth metatarsal bone.

\section{DISCUSSION}

This study was carried out to reveal the prevalence of fibularis tertius muscle in the African population of the south-western Nigeria. We are reporting 63\% prevalence of the muscle in the studied population. Meanwhile, a study conducted on cadavers by Tixa [6] in 1979, reported the prevalence of fibularis tertius muscle (FTM) among a black population to be $86 \%$, although they did not specify if their subjects were from an African population. Their findings present a sharp contrast to what is herein reported. To the best of our knowledge, this work presents the first data on the prevalence of FTM within an African population. This work has helped establish that FTM prevalence is greater on the right limb (67\%) than on the left limb (58\%), as seen in Table 1. However, gender disparity in its right-left occurrences has often been reported [4,9] aligning with our current observa- tions in Table 2 and Figure 2.

Part of the evaluation technique used in this study was recommended by Ramirez et al. [9] and was found to be reliable by Tixa [6] and Kendall et al. [8]. With the further modification of their techniques, it was possible to evaluate the location of the fibularis tertius tendon in all our subjects. FTM was found in this work to course in between the tendon of the last extensor digitorum longus and the bulging of the extensor digitorum brevis. Such surface identification site has not been emphasized in previous literatures but was determinant in all the subjects we examined. It should be noted that the level of accuracy presented by MRI investigations on FTM prevalence is equal to that observed with the kind of manual surface palpation engaged in this work [4].

Of the 100 subjects examined, according to Table 3 and Figure 3, 15\% had the muscle on right limbs only, $6 \%$ had it on left limbs only, $52 \%$ had it on both limbs and $27 \%$ of the subjects did not have the muscle on either of their limbs. These findings suggest that in the population studied, FTM has reached about $52 \%$ success in evolution of its right-left simultaneous occurrence. Nonetheless, the remaining $48 \%$ of the population studied that showed its absence on one or both feet buttresses the fact that the muscle might not have reached its final stage of evolution [10]. Moreover, in relation to the sex of the population studied, our results showed a greater prevalence of the FTM in males $(66 \%)$ than in females (59.4\%), as seen in Figure 1. This finding correlates with the reports of Ramirez et al. [9] and Witvrouw et al. [4].

Most interestingly, FTM is uniquely associated with the evolution of bipedalism. Jungers et al. [11] had claimed that the appearance of fibularis tertius muscle is closely related to the development and evolution of the extensor digitorum longus muscle because its embryological formation is based on the progressive separation from the extensor digitorum longus muscle, until the final insertion in the lateral border of the foot. The somewhat variant palpation site of the FTM tendon, as observed in this work (in Figure 6), may indicate a slightly different developmental pattern of the muscle in the African population

Various anatomical variations in the insertion pattern of the FTM have been reported $[2,12,13]$. Classical textbooks describe FTM to insert on the base of the fifth metatarsal bone (as in Figure 5). It is often reported to attach beyond this point and sometimes, it reaches the phalanges or it's attached to other neighbouring tendons. But, we observed (in Figure 4), its tendons or aponeuroses were palpable along their way to the mid-body of the fifth metatarsal bone in all the subjects studied and this present a unique region of palpation and/or insertion of this muscle within the studied population. 
As represented in Table 4, subjects without the FTM are not in any way more prone to ankle injuries than those with the muscle. Thus, this research confirms the findings of Witvrouw [4], and has also proven that the absence of fibularis tertius muscle does not put one at a danger of ankle injuries. In addition, Vertullo suggested that the insertion of the muscle might play an important role in the causation of torsional stress as observed in Jones fractures and stress fractures [14]. This may further explain the link between the prevalence of the muscle and injuries.

Literatures have claimed that the functional significance of the fibularis tertius muscle can be well compensated for by other muscles of the leg, when it is absent. Eliot and Jungers also stated that subjects without fibularis tertius muscle recruits fibularis longus and fibularis brevis muscle to evert their feet, therefore the absence of the muscle is fully compensated for, as regards eversion [15]. However, this explains why subjects in this study, without the muscle did not show a manually observable lesser degree of eversion or dorsiflexion. Although, the functional disadvantages that may emanate from the absence of FTM in stance and swing phase of motion have not been previously or presently investigated.

The knowledge of the techniques of palpations, location and prevalence of FTM may be important to clinicians, foot surgeons, demonstrators, and also towards a successful tissue grafting procedures.

\section{ACKNOWLEDGEMENTS}

Late Prof. E.A Caxton-Martins (FASN) gave significant encouragement in the development of this article.

\section{REFERENCES}

[1] Jana, R. and Roy T.S. (2011) Variant insertion of the fibularis tertius muscle is an evidence of the progressive evolutionary adaptation for the bipedal gait. Clinics and Practice, 1, e81. doi:10.4081/cp.2011.e81

[2] Joshi, S.D., Joshi, S.S. and Athavale, S.A. (2006) Morphology of peroneus tertius muscle. Clinical Anatomy, 19, 611-614. doi:10.1002/ca.20243

[3] Das, S., Haji, S.F., Latiff, A., Hiaing, K., Ghafar, A.N. and Othman, F. (2009) Absence of the peroneus tertius muscle: Cadaveric study with clinical considerations. Romanian Journal of Morphology and Embryology, 50,

\section{9-511.}

[4] Witvrouw, E., Borre, K.V., Willems, T.M., Huysmans, J., Broos, E. and De Clercq, D. (2006) The significance of Peroneus tertius muscle in ankle injuries: A prospective study. The American Journal of Sports Medicine, 34, 1159-1163. doi:10.1177/0363546505286021

[5] Larico, I. and Jordan, L. (2005) Frecuencia del musculo peroneo tertius. Revista de Investigación e Información en Salud, 1, 29-32.

[6] Tixa, S. (2006) Atlas de anatomía palpatoria. 2nd Edition, Masson, Barcelona.

[7] Theodorou, D.J., Theodorou, S.J., Kakitsubata, Y., et al. (2003) Fractures of proximal portion of fifth metatarsal bone: Anatomic and imaging evidence of a pathogenesis of avulsion of the plantar aponeurosis and the short peroneal muscle tendon. Radiology, 226, 857-865. doi:10.1148/radiol.2263020284

[8] Eliot, D.J. and Jungers, W.L. (2000) Fifth metatarsal morphology does not predict presence or absence of fibularis tertius muscle in hominids. Journal of Human Evolution, 38, 333-342. doi:10.1006/jhev.1999.0337

[9] Ramirez, D., Gajardo, C., Caballero, P., Zavando, D., Cantín, M. and Suazo, G.I. (2003) Clinical evaluation of fibularis tertius muscle prevalence. International Journal of Morphology, 28, 759-764.

[10] Bhargava, K.N., Sanyal, K.P. and Bhargava, S.N. (1961) Lateral musculature of the leg as seen in hundred Indian Cadavers. Indian Journal of Medical Sciences, 15, 181185.

[11] Jungers, W.L., Meldrum, D.J. and Stern, J.T. (1993) The functional and evolutionary significance of the human peroneus tertius muscle. Journal of Human Evolution, 25, 377-386. doi:10.1006/jhev.1993.1056

[12] Figen, T., Qaiser, S. and Serdar, T. (2009) Coexistence of anomalous $\mathrm{m}$. peroneus tertius and longitudinal tear in the m. peroneus brevis tendon. Joint Diseases and Related Surgery Case Report, 20, 165-168.

[13] Poonam, V., Anterpreet, K.A. and Sudhir, A. (2011) Peroneus tertius an evolutionary appearing muscle: A case report. Journal of Life Sciences, 3, 97-99.

[14] Figen, T., Qaiser, S. and Serdar, T. (2009) Coexistence of anomalous $\mathrm{m}$. peroneus tertius and longitudinal tear in the $\mathrm{m}$. peroneus brevis tendon. Joint Diseases and Related Surgery Case Report, 20, 165-168.

[15] Eliot, D.J. and Jungers, W.L. (2000) Fifth metatarsal morphology does not predict presence or absence of fibularis tertius muscle in hominids. Journal of Human Evolution, 38, 333-342. doi:10.1006/jhev.1999.0337 